\title{
Formule semi-empirique pour la détermination de la pression extérieure limite d'instabilité des conduites métalliques lisses noyées dans du béton
}

\author{
A semi-empirical formula for determining \\ the limiting external pressure for the collapse \\ of smooth metal pipes embedded in concrete
}

\author{
PAR R. MONTEL \\ INGÉNIEUn E.C.L. \\ CHEF D'ÉTUDES A LA SOCIÉTÉ DES FORGES E'T ATELIERS DU GREUSOT
}

\begin{abstract}
Se basant sur des notions classiques de résistance des matériaux et utilisant les résultats des essais objet de l'article précédent ainsi que d'autres plus anciens, l'auteur établit une formule pour déterminer la pression limite d'instabilité des blindages non renforcés, compte tenu des défauts de forme et du jeut entre tôle et béton. Cette formule vérifie de façon acceptable les essais récents sur $\varnothing 4100 \mathrm{~mm}$, et ceux effectués antérieurement par $M$. Borot sur $\varnothing 955 \mathrm{~mm}$; elle permet également de calculer des courbes de contrainte en fonction de la pression qui sont en bon accord avec celles relevees au cours des essais. Des essais supplémentaire sur modèle réduit $\varnothing 200 \mathrm{~mm}$ confirment ces résultats et permettent de fixer des limites de validité qu'il convient de respecter en raison de la nature semi-empirique de la formule proposée.
\end{abstract}

\begin{abstract}
Using classical strength of materials techniques together with the results of the tests described in the previous article and the results of earlier tests, the author derived a formula for the limiting collapse pressure for unreinforced linings, which allows for shape defects and for the clearance between the concrete and the plating. This formula satisfactorily checks the recent tests on $4,100 \mathrm{~mm}$ diameter pipes as well as those performed bij Borot on $955 \mathrm{~mm}$ diameter pipes. It also enables stress versus pressure curves to be drawn which check well with those obtained during the tests. Further tests on a $200 \mathrm{~mm}$ diameter model verified the results and enabled limits to be set within which the formula could be used, this being necessary because of its semi-empirical nature.
\end{abstract}

On a vu dans l'article précédent que les méthodes de calcul actuelles ne paraissent pas rendre compte de façon satisfaisante d'essais avec jeu et défauts de forme.

Or, les essais montrent que les défauts de forme et le jeu sont des facteurs importants et l'expérience a déjà permis de constater (Kemano, Roselend, etc.) que, quel que soit le soin pris pour le blocage, le jeu existe du fait des écarts thermiques et du fluage lent du rocher sous la charge que lui transmet la conduite lorsqu'elle est en pression.

L'établissement d'une théorie complète est donc souhaitable. Bien qu'il semble a priori très ardn d'introduire les défauts de forme, le fait que leur répartition n'est pas quelconque per- 
met d'espérer une issue dans cette voic. Il est également encourageant de constater que ces défauts, ou tout au moins les plus importants d'entre eux, ont un profil très voisin de celui des déformations que l'on observe lors de la mise sous pression extérieure.

Une telle théorie implique cependant de sérieuses complications et difficultés mathématiques. En outre, les impondérables et les facteurs aléatoires (difficulté à réaliser des déterminations précises des défauts de forme et du jeu, inégalité de leur répartition, etc.) laisseront toujours subsister une marge importante d'incertitude sur la valeur pratique de la pression d'instabilité, incertitude qu'il faudra compenser par un coefficient de sécurité lui-même important.

Dans ces conditions, il nous a paru opportun de rechercher, à la lumière des indications rechieillies au cours des essais, une formule semiempirique simple susceptible de guider le projeteur en attendant la mise au point d'une théorie mathématique complète.

\section{Etablissement.}

On prend en considération les facteurs suivants susceptibles d'intervenir sur la pression d'instabilité $\mathbf{P}_{i}$ :

- Rayon du tuyau........... R

- Epaisseur du tuyau.........e

— Limite élastique.......... $\sigma e$

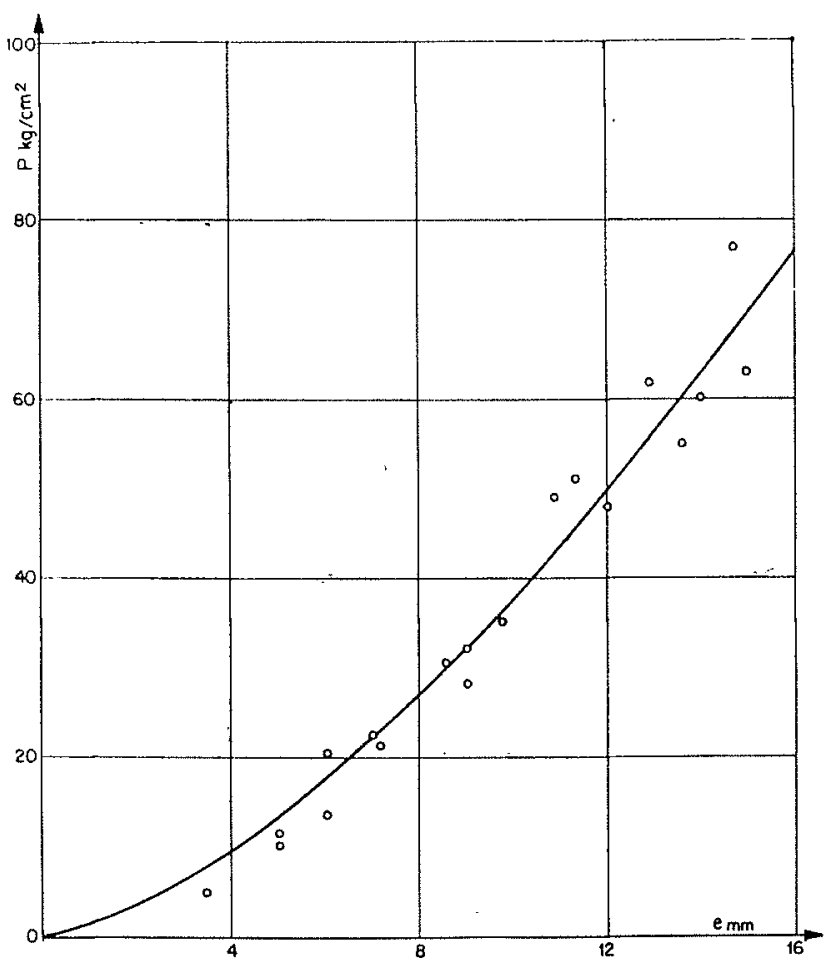

FIG. 1
- Amplitude de l'écart de forme $u$

- Nombre d'onde........... $n$

- Jeu radial. .............. $j$

EPAISSEUR ET RAYON :

Le phénomène est la superposition d'une compression et d'une flexion. Il faut donc s'attendre à ce que l'épaisseur intervienne par une combinaison de sa première et sa deuxième puissances, et même de puissances supérieures puisque les déformations ne sont pas négligeables. Mais il n'est pas exclu que, dans un certain champ assez réduit d'épaisseur, cette combinaison puisse se ramener à une puissance unique sans erreur importante.

En considérant la figure 1 qui représente, en fonction de l'épaisseur, les résultats des 19 essais publiés par M. Borot $\left(^{*}\right)$, on constate qu'il est possible de tracer une courbe continue par rapport à laquelle les points figuratifs ne présentent que des écarts assez faibles. Si ces écarts sont imputés aux variations des paramètres autres que l'épaisseur, cette courbe moyenne rend compte de l'influence de l'épaisseur, abstraction faite des autres facteurs.

La forme: $\mathrm{P}_{i}=f\left(e^{1,5}\right)$ paraît satisfaisante.

Il est généralement admis que le rayon et l'épaisseur interviennent, par leur rapport, avec la notation $\mathrm{R} / e=\mathrm{K}$; nous sommes donc amenés à :

$$
\mathrm{P}_{i}=f\left(\frac{1}{\mathrm{~K}^{1,5}}\right)
$$

DÉfauts DE FORME :

Timoshenko (Résistance des matériaux, II, $\$ 41$ ) a montré que le moment de flexion dans un anneau libre soumis à la pression extérieure était :

$$
\mathrm{M}=p \mathbf{R} u
$$

La contrainte de flexion est ainsi :

$$
\mathrm{F}=\frac{6 p \mathrm{R} u}{e^{2}}=6 p \frac{\mathrm{R}}{e} \frac{u}{e}
$$

L'épaisseur et le rayon intervenant par leur rapport, le défaut de forme intervient par son rapport avec l'épaisseur.

La figure 2 représente, en fonction de l'épaisseur, les résultats de nos cinq essais destructifs, sans jeu. Nous avons tracé la courbe:

$$
\mathrm{P}_{i}=f\left(e^{1,5}\right)
$$

qui passe par le point figuratif de l'essai du premier tronçon de $12 \mathrm{~mm}$, c'est-à-dire la courbe :

$$
\mathrm{P}_{i}=5,8\left(\frac{e}{12,2}\right)^{1,5}
$$

(*) La Houille Blanche, $\mathrm{n}^{\circ}$ 6, 1957, pp. 881-887. 
Fig. 2

Résultats d'essais et influence des défauts de forme.

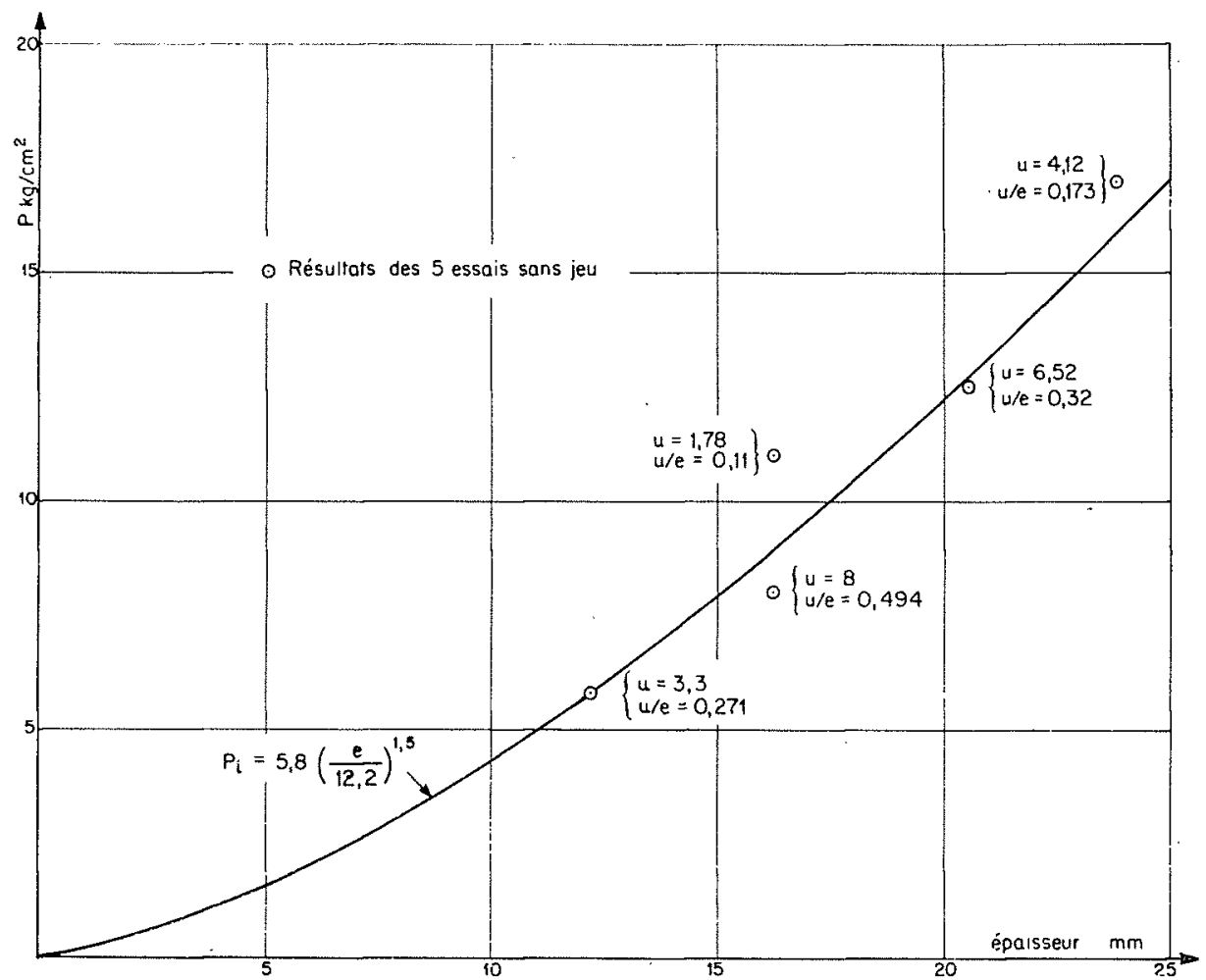

A côté des points figuratifs des essais, sont indiqués l'écart de forme radial « $u »$ et le rapport $u / e$.

Comme exposé précédemment, l'écart de forme considéré est la moyenne des écarts mesurés au gabarit de $50^{\circ}$ aux cinq étages de la génératrice situés au sommet de l'onde.

La courbe correspondant au tronçon de $12 \mathrm{~mm}$ est donc relative à $u / e=0,271$; on voit que les essais comportant des rapports inférieurs à cette valeur se placent au-dessus de cette courbe et les autres au-dessous; de plus, les distances à la courbe se classent dans le même ordre que le rapport $u / e$ :

- en dessous : le point 0,32 est très près de la courbe 0,271 ; le point 0,494 est plus éloigné,

-.. en dessus : le point 0,173 est plus près que le point 0,11 .

Par contre, le classement par les « $u »$ ne scrait pas satisfaisant puisque le point de $24 \mathrm{~mm}$ avec $u=4,12 \mathrm{~mm}$ est au-dessus de la courbe passant par le point $12 \mathrm{~mm}$ avec $u=3,3 \mathrm{~mm}$.

Nous admettrons donc:

$$
\mathrm{p}_{i}=f\left(\frac{1}{u / e}\right)
$$

D'autre part, dans le cas de l'annean libre traitć par Timoshenko, l'expression précédente permet d'écrire celle de la contrainte totale :

$$
\sigma=\frac{p \mathrm{R}}{e}+6 p \frac{\mathrm{R}}{e} \frac{u}{e}=\frac{p \mathrm{R}}{e}\left(1+\frac{6 u}{e}\right)
$$

d'où :

$$
p=\frac{\sigma}{\mathrm{K}} \frac{1}{1+(6 u / e)}
$$

Nous cherchons donc, dans le cas des tuyaux bétonnés, à utiliser la forme :

$$
\mathrm{P}_{i}=f\left(\frac{1}{1+\mathrm{A}(u / e)}\right)
$$

La comparaison des résultats d'essai et des courbes de contraintes 16 -I et 16 -II montre que la valeur: $\mathrm{A}=1,2$ est satisfaisante. Elle donne en effet, pour le deuxième essai en partant du premier :

$$
\mathrm{P}_{i}=11(1,132 / 1,593)=7,82 \mathrm{~kg} / \mathrm{cm}^{2}
$$

soit un écart de l'ordre de $2 \%$ avec la valeur de $8 \mathrm{~kg} / \mathrm{cm}^{2}$. Ceci nous amène donc à la formule :

$$
\mathrm{P}_{i}=f\left(\frac{1}{\mathrm{~K}^{1,5}[1+1,2(u / e)]}\right)
$$

NOMBRE D'ONDES :

Les théories de MM. Amstutz, Borot et Juillard font appel à la notion de raccourcissement exposée précédemment $\left(^{*}\right)$; elles utilisent l'hypothèse suivant laquelle la longueur développée totale du tuyau est égale à celle qui résulte de la seule compression simple due à la pression extérieure; au-

(*) La Houille Blanche, $\mathrm{n}^{\circ} 4$, juin 1960. 
trement dit, le raccoureissement de l'onde compense exactement celui que devrait prendre la tôle restée au contact du béton. Dans ces condiLions, si le nombre d'ondes augmente, il y a moins de tôle en contact avec le béton, donc moins de raccourcissement à compenser; comme cette compensation doit en outre se faire par un nombre d'ondes plus grand, la déformation de celles-ci, donc la contrainte qui y règne, seront réduites. De ce fait, la pression d'instabilité doit augmenter quand le nombre d'ondes augmente; elle est fonction du nombre d'ondes.

Mais nous avons vu que les écarts entre pressions calculées et pressions d'essai ne paraissaient pas varier en fonction du nombre d'ondes comme l'exigerait cette hypothèse.

En outre, pour l'essai $16-\mathrm{II}$, le raccourcissement de l'onde unique était supérieur à celui résultant de la compression simple de toute la circonférence, ce quí infirme l'hypothèse ci-dessus.

D'autre part, dans le tableau I, les cinq tronçons non renforcés, essayés sans jeu, sont classés par ordre d'écarts de forme croissants et le nombre d'ondes observé pour chacun d'eux est indiqué.

\section{TABLEAU I}

\begin{tabular}{|c|c|c|c|c|c|}
\hline Désignation & $16-\mathrm{I}$ & $24-\mathrm{II}$ & $12-\mathrm{I}$ & $20-\mathrm{II}$ & $16-\mathrm{II}$ \\
\hline ule & 0,11 & 0,173 & 0,271 & 0,32 & 0,494 \\
\hline Nombre d'ondes & 4 & 4 & 3 & 3 & 1 \\
\hline
\end{tabular}

On voit que le nombre d'ondes a varié en raison inverse de l'écart de forme relatif.

Ces constatations nous ont suggéré l'interprótation suivante :

- C'est parce qu'il existe un défaut de forme initial que la pression produit une déformalion. Il paraît alors logique que l'amplitude de cette déformation soit fonction de la valeur du défaut. Le raccourcissement de l'onde, qui est une conséquence de la déformation, dépend done de la valeux du défaut initial.

- Si les défauts sont faibles, leurs déformations le seront également, ainsi que les raccoureissements qui en résulteront, de sorte que plusieurs ondes devront se développer pour compenser au total le non-raccourcissement de la tôle restée au contact du béton. Plus la valeur des défauts initiaux sera élevée, plus se réduira le nombre d'ondes nécessaires pour assurer la compensation. La valeur d'un défaut initial peut ètre assez élevéc pour que le raccourcissement de l'onde correspondante soil égal, on même supérieur, à celui résultant de la compression simple de toute la circonférence, et seule cette onde peut alors se déveIopper, comme cela s'est produit pour l'essai 16-II.

Dans ces conditions, le nombre d'ondes est fonction de la valeur des écarts de forme ef il doit suffire de tenir compte de ces derniers dans la détermination de la pression d'instabilité. Nous n'introduirons done pas le nombre d'ondes dans la formule cherchee.

\section{LIMITE FLASTIQUE :}

Les essais ont montré que l'instabilité se manifestait lorsque la contrainte totale atteignail la limite élastique. Pour introduire celle-ci et obtenir une formule homogène à partir de (2), nous poserons :

$$
\mathrm{P}_{i}=\frac{\mathrm{B} \cdot \sigma e}{\mathrm{~K}^{1,5}[1+1,2(u / e)]}
$$

En appliquant cette formule aux mèmes essais 16-I et 16-II on constate qu'il faut prendre:

$$
\mathrm{B}=5 \text {. }
$$

La formule pour les essais sans jeu s'écrit donc :

$$
\mathrm{P}_{i}=\frac{5 . \sigma e}{\mathrm{~K}^{1,5}[1+1,2(u / e)]}
$$

\section{INTRODUCTION DU JEU :}

Nous avons constaté au cours des essais que le profil des ondes de déformation résultant de la mise en pression répondait assez bien à l'équalion proposée par M. Borot (voir figure 3 ).

$$
y=\frac{y_{0}}{2}\left(1+\cos \frac{n x}{\mathrm{R}}\right)
$$

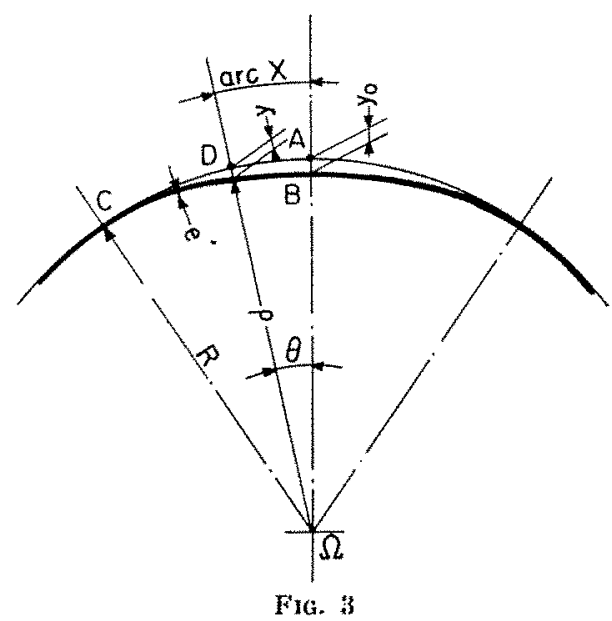


Tableau II

\begin{tabular}{|c|c|c|c|c|c|c|c|}
\hline $\begin{array}{c}\mathrm{P} \\
\mathrm{mm}\end{array}$ & $\begin{array}{c}e \\
\mathrm{~mm}\end{array}$ & $\begin{array}{c}j \\
\mathrm{~mm}\end{array}$ & $\frac{u l}{e}$ & $\begin{array}{c}\sigma e \\
\mathrm{~kg} / \mathrm{mm}=\end{array}$ & $\begin{array}{c}\text { P formule } 5 \\
\mathrm{~kg} / \mathrm{cm}^{2}\end{array}$ & $\begin{array}{l}\text { P essai } \\
\text { lig/cin"- }\end{array}$ & Ecart \\
\hline 2.062 & 12,2 & 0 & 0,271 & 34 & 5,84 & 5,8 & $+0,7 \%$ \\
\hline 2.066 & 16,2 & 0 & 0,11 & 35 & 10,7 & 11 & $-2,7 \%$ \\
\hline 2.066 & 16,2 & 0 & 0,494 & 37 & 8,07 & 8 & $+0,9 \%$ \\
\hline 2.070 & 20,5 & 0 & 0,32 & 32 & 11,4 & 12,5 & $-8,8 \%$ \\
\hline 2.074 & 23,8 & 0 & 0,173 & 34 & 17,3 & 17 & $+1,7 \%$ \\
\hline 2.066 & 16,3 & 1,2 & 0,128 & 35 & 9,25 & 9 & $+2,8 \%$ \\
\hline 2.066 & 16,2 & 0,8 & 0,091 & 35 & 9,9 & 8 & $+23,8 \%$ \\
\hline 479 & 3,5 & 1,1 & 0,1 & 27 & 4,5 & 4,8 & $-6.3 \%$ \\
\hline 479 & $\overline{5}$ & 1,2 & 0,1 & 30 & 9,4 & 10 & $-6 \%$ \\
\hline 479 & 5 & 0,9 & 0,1 & 31 & 10,65 & 11,5 & $-7,3 \%$ \\
\hline 479 & 6 & 1 & 0,1 & 29 & 13,4 & 13,5 & $-0,8 \%$ \\
\hline 478 & 6 & 0,6 & 0,1 & 29 & 15 & 20,5 & $-26,8 \%$ \\
\hline 479 & 7 & 1 & 0,1 & 31 & 18,7 & 22,5 & $-16,9 \%$ \\
\hline 479 & 7,2 & 1 & 0,1 & 30 & 19 & 21 & $-9,5 \%$ \\
\hline 479 & 8,6 & 1,2 & 0,1 & 37 & 27,7 & 30,5 & $-9,2 \%$ \\
\hline 479 & 9 & 0,9 & 0,1 & 33 & 31,3 & 32 & $-2,2 \%$ \\
\hline 479 & 9 & 1 & 0,1 & 25 & 23,3 & 28 & $-16,8 \%$ \\
\hline 479 & 9,8 & 1,1 & 0,1 & 34 & 35,9 & 35 & $+2,5 \%$ \\
\hline 478 & 10,9 & 0,6 & 0,1 & 33 & 45,2 & 48 & $-5,8 \%$ \\
\hline 479 & 11,3 & 1,2 & 0,1 & 38 & 50,1 & 51 & $-1,8 \%$ \\
\hline 479 & 12 & 1,1 & 0,1 & 34 & 50,3 & 48 & $+4,8 \%$ \\
\hline 479 & 12,9 & 1,1 & 0,1 & 37 & 618 & 62 & $-0,3 \%$ \\
\hline 478 & 13,6 & 0,6 & 0,1 & 25 & 48,8 & 55 & $-11,2 \%$ \\
\hline 478 & 14 & 0,5 & 0,1 & 27 & 56,1 & 60 & $-6,5 \%$ \\
\hline 478 & 14,7 & 0,5 & 0,1 & 33 & 73,7 & 77 & $-4,3 \%$ \\
\hline 479 & 15 & 1,2 & 0,1 & 34 & 71,8 & 63 & $+14 \%$ \\
\hline
\end{tabular}


Les principaux écarts de forme observés avant mise en pression ont également une allure très voisine.

M. Borot a montré que la longueur de la demionde correspondant à cette équation est donnée par :

$$
\mathrm{L}=\frac{\pi \mathrm{R}}{n}-\frac{y_{0} \pi}{2 n}+\frac{y_{0}^{2} \pi n}{16 \mathrm{R}}
$$

Pour une onde d'ouverture $60^{\circ}$, ordre de grandeur de ce que nous avons observé, cette expression devient :

$$
\mathrm{L}=\frac{\pi \mathrm{R}}{6}-\frac{y_{0} \pi}{12}+\frac{3 \pi y_{0}^{2}}{8 \mathrm{R}}
$$

Pour les valeurs de $y_{0}$ de l'ordre de 2 à $10 \mathrm{~mm}$, relevées au cours des essais avec un rayon de $2000 \mathrm{~mm}$, le troisième terme est négligeable devant chacun des deux autres et devant leur différence, de sorte que l'on peut écrire sans erreur importante :

$$
\mathrm{L}=\frac{\pi \mathrm{R}}{6}-\frac{y_{0} \pi}{12}
$$

qui exprime la longueur de la demi-onde à l'état initial sans jeu.

S'il existe un jeu $k j »$ entre tôle et béton, les appuis de l'onde doivent se déplacer d'une quantité « $j$ »avant de toucher le béton et le rayon de référence deviendra $(\mathrm{R}+j)$; pour que la longueur de l'onde ne soit pas modifiée, il taudra que l'écart de forme $y_{0}$ devienne $y_{1}$, tel que :

$$
\frac{\pi \mathrm{R}}{6}-\frac{\pi y_{0}}{12}=\frac{\pi(\mathrm{R}+\mathrm{J})}{6}-\frac{\pi y_{1}}{12}
$$

d'oil :

$$
y_{1}=y_{0}+2 j
$$

qui exprime que le jeu a pour effet de majorer le défaut de forme initial. Dans le cas d'un tuyau avec jeu, nous tiendrons donc compte de l'écart de forme $y_{1}$.

Les équations (3) et (4) permettent d'écrire la formule complète:

$$
\mathrm{P}_{i}=\frac{5 . \sigma e}{\mathrm{~K}^{1,5}[1+1,2(u+2 j) / e]}
$$

avec :

$$
\begin{aligned}
\sigma e & =\text { limite élastique }: \mathrm{kg} / \mathrm{mm}^{2} ; \\
\mathrm{K} & =\mathrm{R} / e ; \\
\mathrm{R} & =\text { rayon extérieur : } \mathrm{mm} ; \\
e & =\text { épaisseur : mm } \\
u & =\text { écart radial au gabarit de } 50^{\circ}: \mathrm{mm} ; \\
j & =\text { jeu radial entre tôle et béton }: \mathrm{mm} ; \\
\mathrm{P}_{4} & =\text { pression d'instabilité }: \mathrm{kg} / \mathrm{mm}^{2} .
\end{aligned}
$$

\section{2. - Application aux essais.}

Le tableau II donne, pour nos sept essais destructifs (dont deux avec jeu) et les dix-neuf essais de M. Borot avec jen:

- les conditions d'essai,

- - le résultat d'essai,

- le résultat donné par la formule (5),

- l'écart entre ces deux derniers chiffres.

Pour appliquer la formule aux essais de M. Borot, il était nécessaire de connaître la valeur de l'écart de forme, et celui-ci n'a pas été mesuré. Pour en avoir une valeur approximative, nous avons raisonné comme suit :

Les viroles essayées étaient non pas bétonnèes, mais introduites dans un moule métallique tourné; l'espace entre virole et moule constituait le jeu et il était de l'ordre de 0,5 à $1,2 \mathrm{~mm}$. Pour parvenir à introduire ces viroles de $955,5 \mathrm{~mm}$ de diamètre dans le moule avec un jeu aussi faible, il a certainement été nécessaire de procéder à un calibrage très poussé. Lorsqu'au cours de nos essais, nous avons fait un calibrage très poussé, l'écart de forme qui en est résulté a été voisin de 0,1 e (essais $16-\mathrm{I}, 16-\mathrm{III}$ et $16-\mathrm{IV}$ ); nous avons donc admis, comme ordre de grandeur, que toutes les viroles essayées par M. Borot presentaient cet écart moyen de $0,1 e$.

On voit que :

- Pour nos essais sur $\varnothing 4100 \mathrm{~mm}$, l'écart entre la pression réelle et celle calculée pour la formule (5) est faible pour les cinq cas sans jeu et un cas avec jeu; il est, par contre, important pour le deuxième cas avec jeu (nous avons montré précédemment que le résultat de cet essai était d'ailleurs incompatible avec ceux des autres troncons de $16 \mathrm{~mm})$;

- Pnur l'ensermble des vingt-six cas, dont les dix-neuf sur $\varnothing 956 \mathrm{~mm}$ avec jeu de M. Borot, la répartition des écarts est la suivante:

- écart roisin de $25^{\circ} \%$ dans deux cas, c'està-dire $8 \%$ des essais;

- écart voisin de $15 \%$ dans trois cas, c'està-dire $12 \%$ des essais;

- écart $\leqslant 11 \%$ dans vingt et un cas, e'està-dire $80 \%$ des essais;

- moyenne des écarts pour les vingt-six cas: $-3,7 \%$.

La formule (5) donne donc dans l'ensemble des résultats acceptables. 


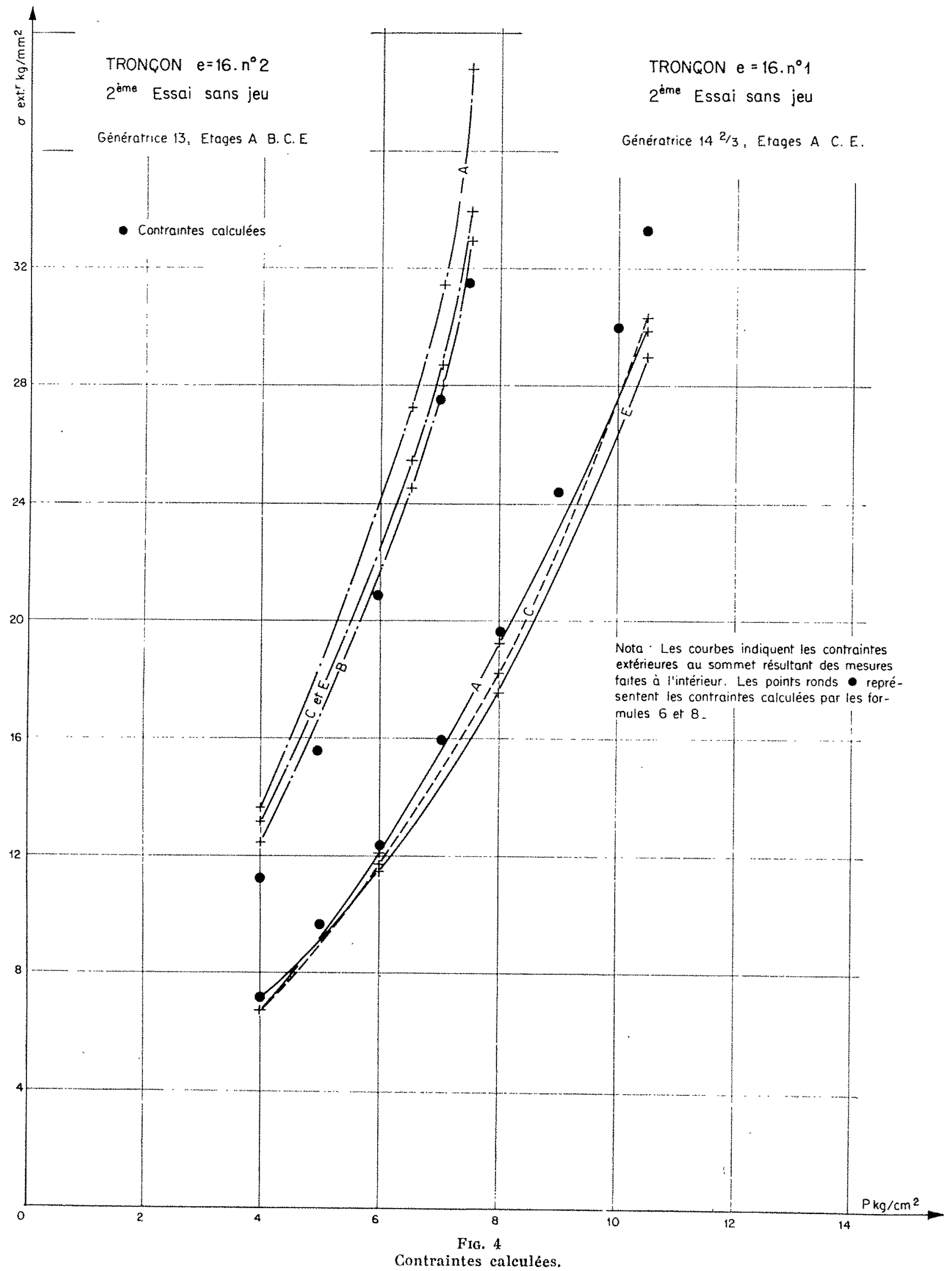


TRONGON $e=20 . n^{\circ} 2$

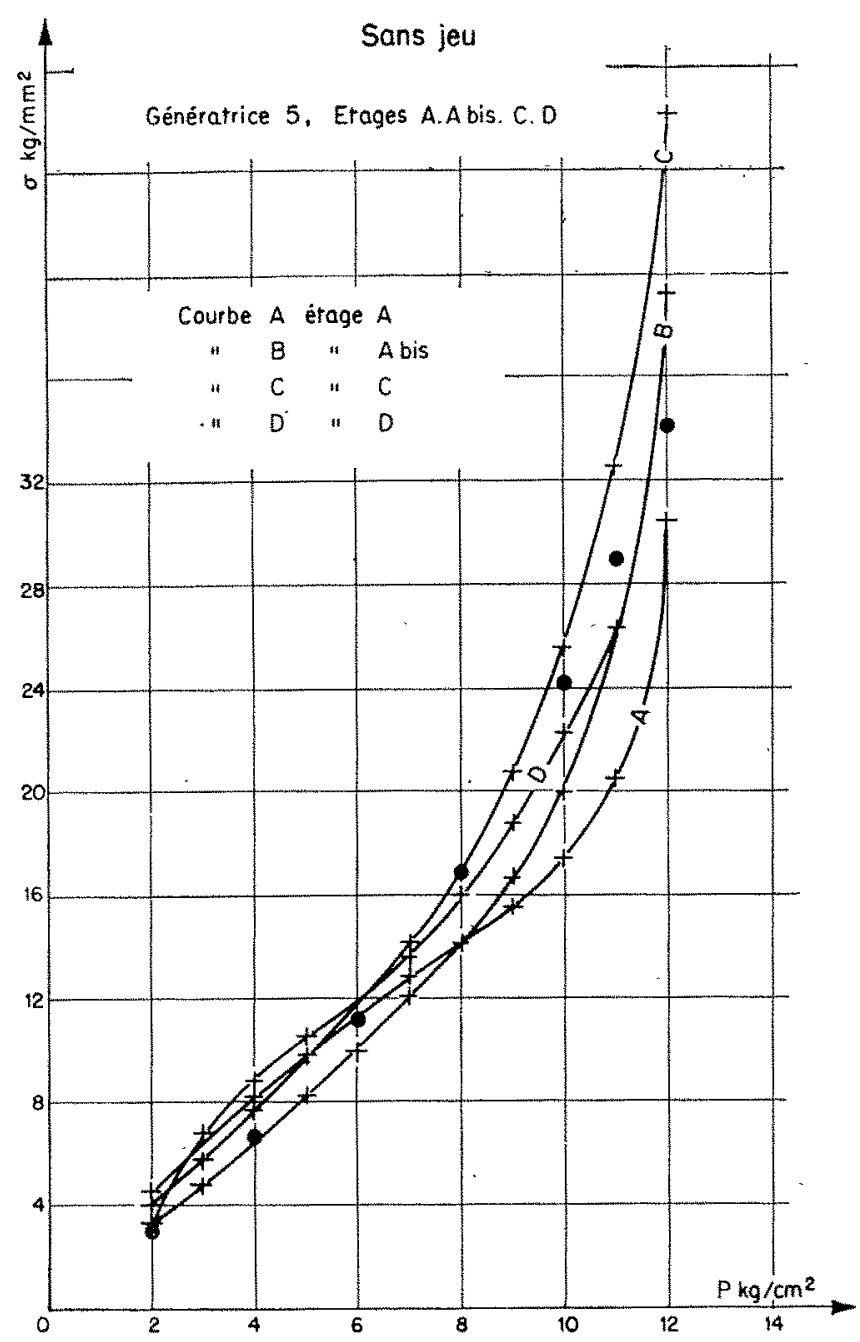

TRONCON e $=16, n^{\circ} 1$

1" Essai avec jeu

Génératrice $142 / 3$, Erages A.C.E

- Contraintes calculées

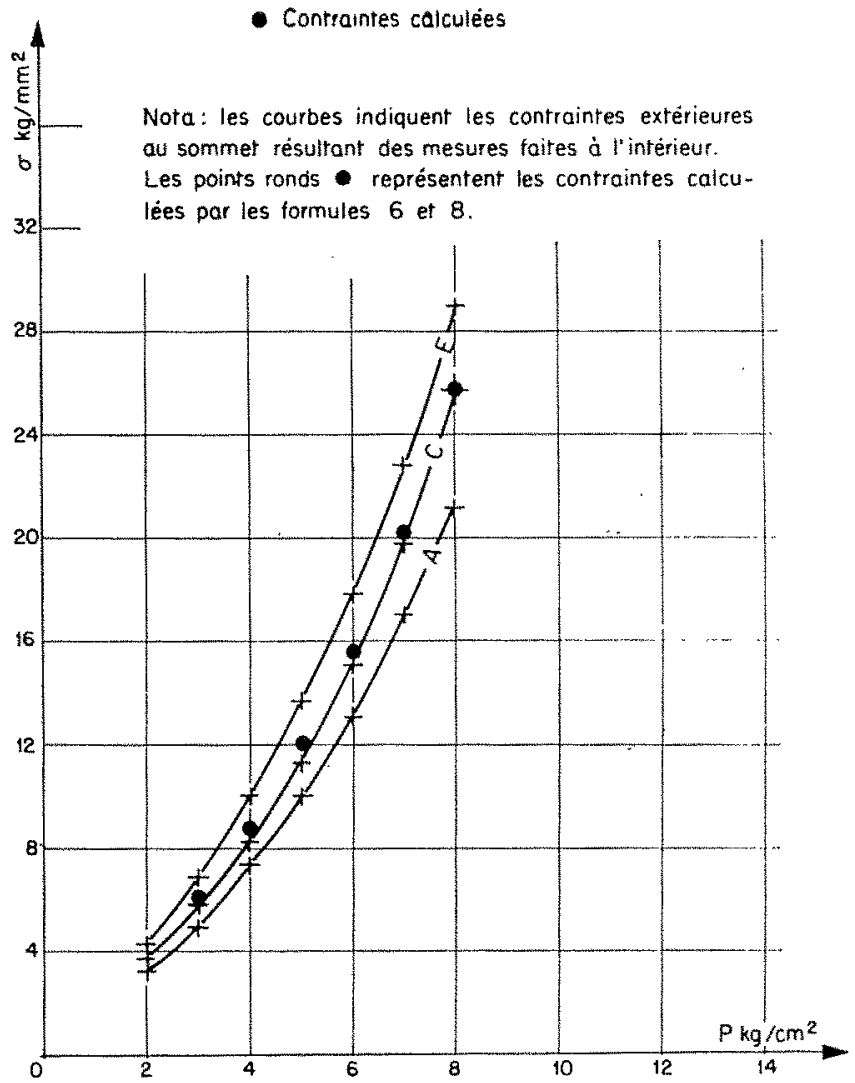

Fil. 4

Contraintes calculées.

\section{3. - Essais sur modèle réduit.}

Afin de vérifier la validité de la formule pour un diamètre nettement différent de $4100 \mathrm{~mm}$ et pour des rapports $K$ faibles, nous avons procédé également à divers essais sur des viroles de diamètre $200 \mathrm{~mm}$ et de $300 \mathrm{~mm}$ de longueur. Elles étaient introduites dans un moule métallique et hétonnées de la même façon que pour les essais en vraie grandeur. Les épaisseurs utilisées ont été de $1,2 \quad 1,5$ et $3 \mathrm{~mm}$.

On mesurait seulement les défauts de forme. Ceux-ci étant très faibles (quelques dixièmes de millimètre), la précision de leur mesure était un peu aléatoire, malgré l'emploi d'un comparateur au centième de millimètre, par suite des défauts de surface des tôles.

Les résultats ont élé en bon accord avec la formule 3, l'écart n'excédant pas 10\%. Pour l'épaisseur $1,2 \mathrm{~mm}$, on a trouvé des pressions d'instabilité de 15 et $16 \mathrm{~kg} / \mathrm{cm}^{2}$ avec une limite élastique de $27 \mathrm{~kg} / \mathrm{mm}^{2}$. Pour l'épaisseur $3 \mathrm{~mm}$, la pression a été de 62 et $67,5 \mathrm{~kg} / \mathrm{cm}^{2}$ pour une limite élastique de $31 \mathrm{~kg} / \mathrm{mm}^{2}$.

Tous les essais précédents ont montré que, dans le domaine étudié, le phénomène était une instabilité plastique, puisque l'instabilité se produisait lorsque la contrainte atteignait la limite élastique. Il est cependant vraisemblable que, pour des valeurs suffisamment élevées de la limite élastique, l'instabilité doit se produire dans le domaine élastique par flambage pur. Afin de rechercher cette limite d'instabilité élastique, nous avons procédé aux trois essais suivants :

- Une virole, ép. $1,5 \mathrm{~mm}, \sigma e=46 \mathrm{~kg} / \mathrm{mm}^{2}$, $u=0,5 \mathrm{~mm}$, qui a péri à $30 \mathrm{~kg} / \mathrm{cm}^{2}$, soit un écart de $-2,2 \%$ avec les $29,3 \mathrm{~kg} / \mathrm{cm}^{2}$ résultant de la formule (5);

$u=0,36 \mathrm{~mm}$, qui a péri à $35 \mathrm{~kg} / \mathrm{cm}^{2}$ alors - Une virole, ép. $1,5 \mathrm{~mm}$, $\sigma e=105 \mathrm{~kg} / \mathrm{mm}^{2}$. 
que la formule (5) domne $73 \mathrm{~kg} / \mathrm{cm}^{2}$; on trouve que la formule donnerait $35 \mathrm{~kg} / \mathrm{cm}^{2}$ pour une limite élastique de $50 \mathrm{~kg} / \mathrm{mm}^{2}$;

- Une virole, ép. $1,4 \mathrm{~mm}, \sigma e=76 \mathrm{~kg} / \mathrm{mm}^{2}$, $u=0,7 \mathrm{~mm}$, qui a péri à $26,6 \mathrm{~kg} / \mathrm{cm}^{2}$ alors que la formule (5) donne $38,6 \mathrm{~kg} / \mathrm{cm}^{2}$. En utilisant la limite élastique fictive de $50 \mathrm{~kg} / \mathrm{mm}^{2}$, la formule donne $25,4 \mathrm{~kg} / \mathrm{cm}^{2}$, c'est-à-dire un écart de $-5^{\circ} \%$ avec la pression réelle.

Dans les deux demiers cas, on a donc eu vraisemblablement le flambage pur, c'est-à-dire formation d'une bosse pour une contrainte inférieure à la limite élastique, et il semble que cette limite de flambage pur peut être déterminée en utilisant dans la formule (5) une limite clastique fictive de $50 \mathrm{~kg} / \mathrm{mm}^{2}$.

Dans ces conditions, pour la détermination des pressions d'instabilité, on ne prendra pas en considération les valeurs de limite élastique supérieures à $50 \mathrm{~kg} / \mathrm{mm}^{2}$.

\section{4. - Application au calcul des courbes de contrainte.}

En posant, pour simplifier l'écriture:

$$
\mathrm{M}=1,2 \frac{u+2 i}{e}
$$

la formule (5) devient:

$$
\mathrm{P}_{i}=\frac{5 . \sigma e}{\mathrm{~K}^{1,5}(1+\mathrm{M})}
$$

qui s'écrit aussi :

$$
\sigma e=\frac{\mathrm{P}_{i} \mathrm{~K}^{1,5}(1+\mathrm{M})}{5}
$$

Cette forme est une expression particulière de la contrainte au moment où l'on atteint la pression d'instabilité $P_{i}$.

La forme :

$$
\sigma=\frac{\mathrm{PK}(1+\mathrm{M})}{1-\left(\mathrm{P} / \mathrm{P}_{i}\right)\left[1-\left(5 / \mathrm{K}^{0,5}\right)\right]}
$$

est une expression de la contrainte $\sigma$ pour une pression quelconque $\mathrm{P}$, qui pour $\mathrm{P}=\mathrm{P}_{i}$ redonne bien l'expression particulière (7).

On remarque que la forme (8) est vicieuse puisque, par l'intermédiaire de $\mathrm{P}_{i}$, elle rend la contrainte $\sigma$ fonction de la limite élastique $\sigma e$. 'loutefois, en faisant des applications numériques, on constate que cette influence de oe sur $\sigma$ est assez faible.

Nous avons donc appliqué les équations et (8) an calcul des courbes de contrainte pour divers essais (16-1, 16-1I, 20-II et 16-I non destructif avec jeu). La figure 4 donne la comparaison avec les contraintes extérieures déduites des mesures effectuées pendant les essais. (Ie calcul par les équations (6) et (8) est basé sur les « conditions d'essai » du tableau 6 de l'article précédent en utilisant « $u »$ moyen).

On voit que, contrairement à toute attente, l'accord est assez bon; donc, malgré son imperfection, l'équation (8) rend compte du déroulement du phénomène. Ceci explique que la formule (5), qui peut s'en déduire, puisse donner avec une assez bonne approximation la pression d'instabilité.

\section{5. - Limites de validité.}

Quoi qu'il en soit, le caractère empirirue de la formule (5) ne doit pas être perdu de vue et il convient de ne pas l'appliquer en dehors du domaine où elle a été vérifiée, c'est-à-dire :

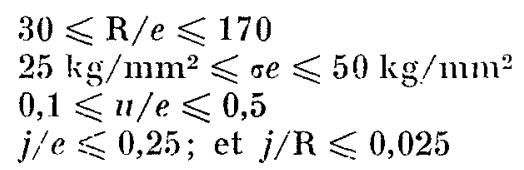

Les limites élastiques supérieures à $50 \mathrm{~kg} / \mathrm{mm}^{2}$ ne doivent pas être prises en considération, car il y a alors risque d'instabilité élastique.

Pour l'écart de forme, il semble que des valeurs de 0,3 à 0,5 puissent être oblenues pratiquement avec un calibrage soigné; un calibrage beaucoup plus poussé a permis d'arriver à 0,1 , mais une telle opération est très onéreuse, les mesures de contrôle deviennent imprécises et il n'est pas exclu que le profil de défauts ramenés à une aussi faible valeur soit plus défavorable que celui d'écarts naturels plus importants.

\section{Conclusion.}

Cette formule semi-empirique simple rend compte de l'évolution des contraintes et permet de retrouver avec une approximation acceptable les résultats de nos essais et ceux de 19 autres essais effectués antérieurement. Il semble done qu'elle puisse dans l'immédiat rendre des services pour la détermination des pressions pratiques d'instabilité sous réserves des limitations impératives inhérentes à sa nature.

Il importe, en effet, de ne pas perdre de vue que dans la meilleure hypothèse elle ne saurait représenter qu'une simplification et une approximation, acceptables seulement dans un domaine restreint, de la solution génćrale. Elle n'a donc pas d'autre prétention que de permettre une transition avec la solution mathématique complète qui reste à établir. 